\title{
Effects of Vestibular Rotatory Accelerations on Covert Attentional Orienting in Vision and Touch
}

\author{
Francesca Figliozzi $^{1,2}$, Paola Guariglia ${ }^{1,2}$, Massimo Silvetti ${ }^{1,2}$, \\ Isabelle Siegler ${ }^{3}$, and Fabrizio Doricchi ${ }^{1,2}$
}

\begin{abstract}
Peripheral vestibular organs feed the central nervous system with inputs favoring the correct perception of space during head and body motion. Applying temporal order judgments (TOJs) to pairs of simultaneous or asynchronous stimuli presented in the left and right egocentric space, we evaluated the influence of leftward and rightward vestibular rotatory accelerations given around the vertical head-body axis on covert attentional orienting. In a first experiment, we presented visual stimuli in the left and right hemifield. In a second experiment, tactile stimuli were presented to hands lying on their anatomical side or in a crossed position across the sagittal body midline. In both experiments, stimuli were presented while normal subjects suppressed or did not suppress the vestibulo-ocular response (VOR) evoked by head-body rotation. Independently of VOR suppression, visual and tactile stimuli presented on the side of rotation were judged to precede simultaneous stimuli pre-
\end{abstract}

sented on the side opposite the rotation. When limbs were crossed, attentional facilitatory effects were only observed for stimuli presented to the right hand lying in the left hemispace during leftward rotatory trials with VOR suppression. This result points to spatiotopic rather than somatotopic influences of vestibular inputs, suggesting that cross-modal effects of these inputs on tactile ones operate on a representation of space that is updated following arm crossing. In a third control experiment, we demonstrated that temporal prioritization of stimuli presented on the side of rotation was not determined by response bias linked to spatial compatibility between the directions of rotation and the directional labels used in TOJs (i.e., "left" or "right" first). These findings suggest that during passive rotatory head-body accelerations, covert attention is shifted toward the direction of rotation and the direction of the fast phases of the VOR.

\section{INTRODUCTION}

Proficient orienting of motor responses in space requires the integration of inputs from different sensory modalities. For instance, combining visual inputs with proprioceptive, vestibular, and reafferent signals arriving from the eye, the head, and the body allows the correct localization of stimuli falling on identical retinal positions even when these are in different spatial locations due to the observer's movements (Crowell, Banks, Shenoy, \& Andersen, 1998; Andersen, Snyder, Bradley, \& Xing, 1997; Galletti, Battaglini, \& Fattori, 1993).

Observers can also attend to positions in space "covertly," without making overt motor responses. As for the case of overt motor orienting, cross-modal integration of afferent inputs facilitates covert orienting, favoring the coordinated gathering of information arriving at the different senses from the spatial location capturing the observer's attention. Several studies have demonstrated that detection of a stimulus in one sensory modality is not only improved by spatial cues from the same

${ }^{1}$ Fondazione Santa Lucia IRCCS, Rome, ${ }^{2}$ Università degli Studi di Roma "La Sapienza," ${ }^{3}$ Université Paris-Sud

modality, but also when inputs from other modalities orient attention toward the stimulus appearance position (Driver \& Spence, 1998). Cross-modal attentional facilitation was found for vision, touch, and hearing (Spence, Nicholls, Gillespie, \& Driver, 1998). Neurons whose discharge is increased by spatially congruent multimodal signals and inhibited by spatially incongruent ones were found in the superior colliculi, the parietal lobes, and the premotor cortex (Bremmer, Schlack, Duhamel, Graf, \& Fink, 2001; Graziano, Hu, \& Gross, 1997; Wallace, Meredith, \& Stein, 1992; Meredith \& Stein, 1986).

With the exception of one experiment using caloric vestibular stimulation (i.e., water irrigation of the auditory canal) reported in a study by Rorden, Karnath, and Driver (2001), systematic investigations on the influence of vestibular signals on covert orienting of spatial attention are lacking, notwithstanding the existence of close functional links between vestibular information and the neural coding of space. Vestibular cues contribute to updating body position after rotations or translations performed in the absence of visual input (Berthoz, 1997). They also allow the correct interpretation of optic flow changes as being due to active changes in the observer's position with respect to the environment or 
vice-versa (Bremmer, et al., 2001; Berthoz, 1997). The influence of vestibular signals on space coding is also seen in the modifications of the subjective position of stimuli presented during rotatory accelerations around the vertical body axis. Rotations in one horizontal direction shift the perceived position of stimuli aligned with the head-body midsagittal plane toward the counterrotatory direction (Clark \& Graybiel, 1949; Graybiel \& Hupp, 1946). These illusory effects have been described for both visual (oculogyral illusion) and acoustic (audiogyral illusion) stimuli. Caloric vestibular and rotatory stimulations also displace the subjective auditory midsagittal plane (the so-called "straight ahead") in the direction opposite to that of head-body rotation (Lewald \& Karnath, 2000, 2001).

The aim of the present study was to investigate the influence of dynamic variations of vestibular input due to rotatory accelerations around the vertical head-body axis on lateral covert attentional orienting in the visual and tactile modalities. We specifically wished to determine whether rotatory accelerations bias attention toward or away from the left or right side of rotation. This should provide novel and important information on how body motion and visuospatial processing interact, extending the study of covert attentional orienting from intensively explored experimental conditions in which head-body position is kept constant to poorly explored conditions in which attentional shifts are captured and measured during eye, head, or body motion (see, for example, Vuilleumier \& Schwartz, 2001).

We compared attentional performance in three different vestibular conditions: motionless-baseline (i.e., no vestibular stimulation), leftward rotatory accelerations, rightward rotatory accelerations. In each of these conditions, bias of covert attention toward the left or the right side of space was measured with a "temporal order judgment" task (TOJ; Rorden, Karnath, \& Driver, 2001; Stelmach \& Herdman, 1991). In this task, two temporally simultaneous or asynchronous stimuli are presented in each trial (one to the left and one to the right of the head-body midsagittal plane) and subjects are forced to judge which one of the two stimuli "came first." Several studies have demonstrated that shifting attention toward a spatial location, whether reflexively or voluntarily, favors earlier conscious detection (i.e., prior entry) of stimuli at the attended location compared with temporally simultaneous stimuli presented at an unattended position (see, for a short review of the literature, Shore, Spence, \& Klein, 2001). Therefore, when a left and a right stimulus are simultaneously presented, leftward shifts of attention should increase the frequency of "left-first" responses and rightward shifts the frequency of "right-first" responses compared with a neutral attentional baseline.

In a first experiment, we examined the effects of rotatory vestibular stimulation on covert visual attention. In a second experiment, we investigated the same effects on covert orienting of attention in the tactile modality. In the second experiment, the subjects' hands were positioned on their anatomical side or crossed across the head-body midsagittal plane. These two conditions served to investigate whether vestibular inputs influence covert tactile orienting according to somatosensory coordinates (i.e., the side of the body the hand is anatomically connected to) or spatiotopic ones (i.e., the side of space where the hand is positioned). Finally, in a third experiment, we checked the influence of "response bias" on vestibular attentional facilitation. In the present study, subjects might tend to report as "first come" the stimulus presented on the side of rotation simply due to the fact that the spatial code engaged by the direction of rotation biases subjects to respond with a spatially compatible directional label. We evaluated the role of response bias using a task in which vestibular directional cueing and response dimensions were orthogonal (Spence et al., 2001; Cairney, 1975; Drew, 1896). One stimulus of the pair appeared on one lateral side above or below a short horizontal line segment and the other stimulus on the opposite lateral side and opposite vertical location with respect to another horizontal line segment. Subjects had to decide whether the stimulus appearing first was the one above or below the segment ("up-first" or "down-first" decision rather than "left-first" or "right-first" decision).

Rotations around the vertical body axis in total darkness typically elicit a vestibular-ocular reflex (VOR) consisting of slow horizontal eye movements in the counter-rotatory direction alternating with rapid eye movements in the rotatory direction (Leigh \& Zee, 1999; Siegler, Israël, \& Berthoz, 1998). The VOR can be actively suppressed when a fixation point moves with the experimental subject (Leigh \& Zee, 1999; Siegler et al., 1998; Israël, Bronstein, Kanayama, Faldon, \& Gresty, 1996). This procedure ideally allows for evaluating the influence of rotatory accelerations on attentional orienting, either in the presence or in the absence of the reflexive eye displacements caused by vestibular stimulation. Therefore, in the present study, rotatory accelerations were given in two different oculomotor conditions. In the first condition (no VOR suppression), central fixation was absent and the release of VOR allowed. In the second condition (VOR suppression), subjects were required to suppress the VOR by maintaining their gaze on a central fixation reference aligned to the head-body midsagittal plane, which remained on for the entire duration of the rotatory movement.

\section{RESULTS}

\section{Experiment 1: Vestibular-Visual Prior Entry}

Angular transformation of individual percentages of "left-first" responses were submitted to a Vestibular condition (motionless baseline, leftward rotation, right- 
ward rotation) $\times$ VOR suppression (present, absent) within-subjects ANOVA. Only a main vestibular condition effect was found $[F(2,20)=17, p<.001$; see Figure 1], with an increment in the frequency of "left-first" responses during leftward rotations (63.7\%) and a decrement of the same responses during rightward rotations (28\%) compared with motionless baseline (41.7\%). Performance in the different VOR suppression conditions was further explored through planned mean comparisons. With VOR suppression, leftward accelerations increased the frequency of "left-first" responses compared to motionless baseline, whereas rightward accelerations decreased the frequency of "left-first" responses (all comparisons $p<.05$ ). Without VOR suppression, leftward rotations increased the frequency of "left-first" responses compared to motionless baseline $(p<.01)$. During rightward rotations, there was a trend toward reduced frequency of "left-first" responses $(p=.1)$. Percentage differences normalized to motionless results are reported in Figure 1. Percentages of "leftfirst" responses to asynchronous trials, calculated in the whole sample and across the two VOR suppression conditions, are reported in Figure 1. At short asynchro- nies (from -45 to $+45 \mathrm{msec}$ ), rotatory accelerations produced directional biases similar to those observed on synchronous trials. Vestibular influences were not observed at longer asynchronies, where performance was perfect in all vestibular conditions.

Spatial compatibility effects (i.e., faster manual responses for the hand-button on the side of rotation; Umiltà \& Nicoletti, 1990) were investigated to assess the influence of response motor bias (i.e., the tendency to select the motor response on the side of the attentional shift; Shore, Spence, et al., 2001) on TOJ. Averaged individual manual reaction times to simultaneous stimuli were entered in a Vestibular condition (motionless baseline, leftward rotation, rightward rotation) $\times$ VOR suppression (present, absent) $\times$ Hand button side (left, right) within-subjects ANOVA. The ANOVA showed no interaction of hand button side with either of the other two factors $(p>.2)$, demonstrating no influence of spatial compatibility effects on attentional bias for stimuli presented on the rotation side. Typical of unspeeded tasks, manual reaction times were extremely slow (left button: mean $1314 \mathrm{msec}$, SD $674 \mathrm{msec}$; right button: $1194 \mathrm{msec}$, SD $548 \mathrm{msec}$ ). These results are in keeping
Figure 1. Experiment 1: Vestibular-visual prior entry. (A) Percentage of "left-first" responses to synchronous trials in the three rotatory conditions and the VOR and no-VOR suppression conditions. Vertical bars inside line graphs indicate 95\% confidence interval (Loftus \& Masson, 1994). Percentage differences normalized to motionless results are reported inside the box $(\mathrm{MB}=$ motionless baseline; LR = leftward rotation; $\mathrm{RR}=$ rightward rotation). (B) Percentage of "left-first" responses to asynchronous trials (ordinate) plotted against asynchronies (abscissa). Negative asynchronies indicate "first left-second right" trials, positive asynchronies "first right-second left" ones.

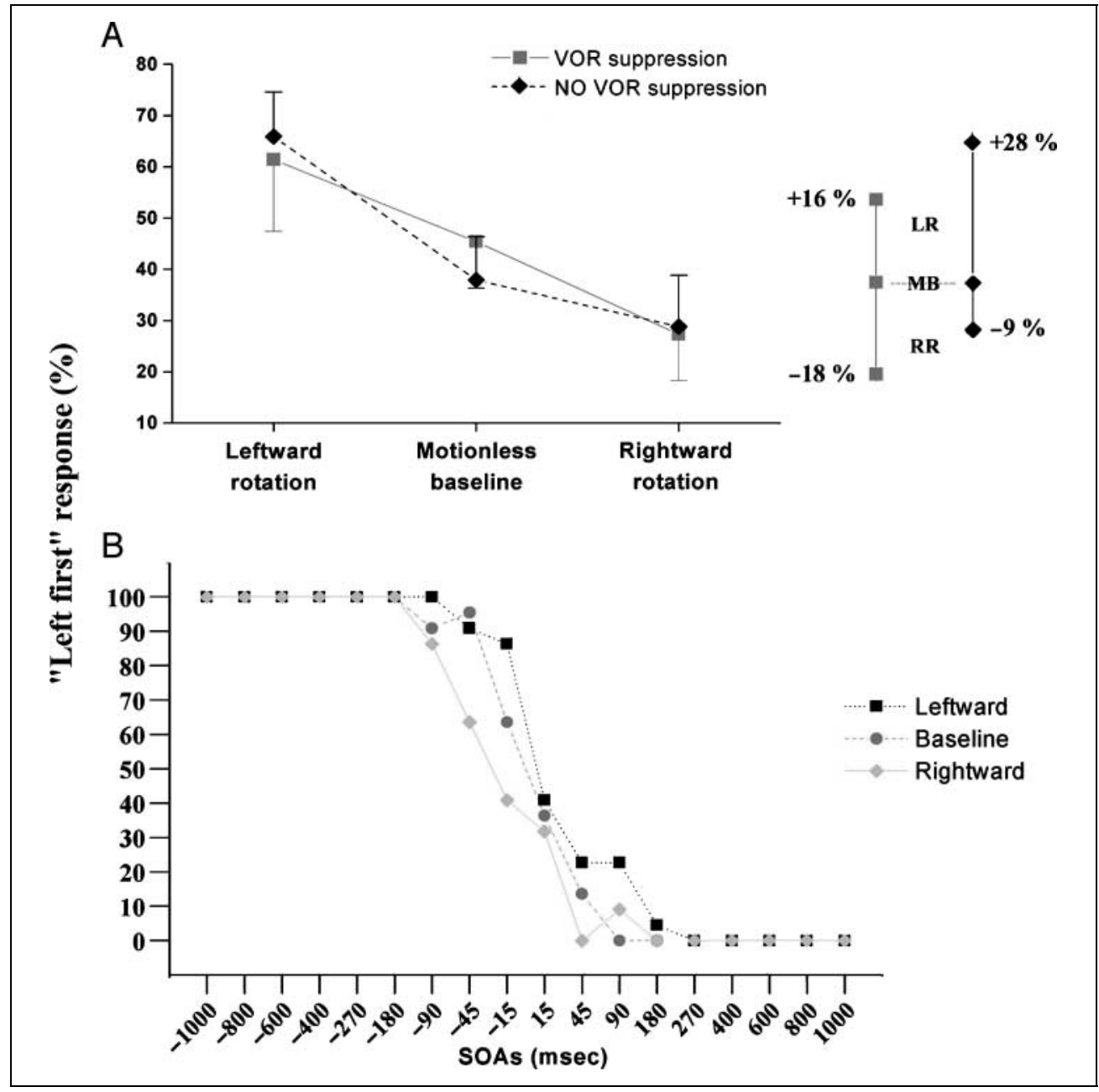


with the well-established absence of spatial compatibility effects for reaction times higher than 500 msec (Umiltà \& Nicoletti, 1990) and confirm that subjects paid attention to accuracy rather than velocity of response.

These results suggest that rotatory acceleration around the vertical head-body axis induces shift of covert visual attention toward the direction of rotation.

\section{Experiment 2: Vestibular-Tactile Prior Entry}

Compared with the first experiment, one additional experimental manipulation was used. The first condition replicated Experiment 1: The subjects' hands were positioned on their anatomical side (i.e., aligned to their shoulders: uncrossed condition). In a second experimental condition, the right hand was positioned in the left egocentric space and the left hand in the right egocentric space (crossed condition). In both conditions, hands were placed on a horizontal panel secured to the rotating chair (see Methods section).

Angular transformation of individual percentages of "hand on left side first" (i.e., "left first") responses were submitted to a Vestibular condition (motionless baseline, leftward rotation, rightward rotation) $\times$ VOR suppression (present, absent) $\times$ Hand position (anatomical, crossed) within-subjects ANOVA. The ANOVA showed a significant main effect for vestibular condition $[F(2,20=$ $16, p<.001]$ and significant Vestibular condition $\times$ Hand position $[F(2,20)=9.7, p=.001]$ and Vestibular condition $\times$ VOR suppression $\times$ Hand position $[F(2,20)=4.2$, $p<.05]$ interactions (see Figure 2). Planned comparisons showed that with hands in anatomical position, leftward accelerations increased the frequency of "leftfirst" responses as compared with motionless baseline, whereas rightward accelerations decreased the frequency of "left-first" responses (all comparisons $p<.01$ ). The attentional bias in the direction of rotation was present independently of VOR suppression (all comparisons $p<.05)$. Percentages of "left-first" responses to asynchronous trials in the anatomical condition, calculated in the whole sample and across the two VOR suppression conditions, are reported in Figure 2. As in Experiment 1, vestibular influences similar to those observed in synchronous trials were observed only at short asynchronies. At longer asynchronies performance was perfect. In the hand-crossed condition, when VOR was not suppressed no variation in the frequency of "left-first" responses was found among the different vestibular conditions (all comparisons $p=n s$ ). With hands crossed and VOR suppression, compared to motionless baseline the frequency of "left-first" responses increased only for stimuli delivered to the right hand in the left hemispace during leftward turns $(p<.01)$. Percentage differences normalized to motionless results are reported in Figure 2. Percentages of "leftfirst" responses to asynchronous trials in the crossed condition, calculated in the whole sample and across the two VOR suppression conditions, are reported in Figure 2. Interestingly, plotting percentages against asynchronies closely reproduced findings reported by Yamamoto and Kitazawa (2001) showing that at moderately short asynchronies (<300 msec), crossing the arms causes inverting of TOJ. As in the study by Yamamoto and Kitazawa, perfect or almost-perfect performance (80-100\% correct TOJ) was observed only at the longest asynchronies ( -1000 and $+1000 \mathrm{msec})$.

Averaged individual reaction times to simultaneous stimuli were submitted to a Vestibular condition (motionless baseline, leftward rotation, rightward rotation) $\times$ VOR suppression (present, absent) $\times$ Hand position (anatomical, crossed) $\times$ Hand button side (left, right) within-subjects ANOVA. The ANOVA showed no significant main effect or interaction, demonstrating no stimulus-response spatial compatibility effects. As in Experiment 1, manual reaction times were slow (left button: $1214 \mathrm{msec}$, SD 676; right button: $1154 \mathrm{msec}$, SD 479).

These results suggest that with hands in anatomical position, rotatory acceleration facilitates shifts of covert tactile attention towards the hand lying on the side of rotation. These facilitatory effects largely disappear when spatiotopic and somatotopic coding of tactile stimuli are made incongruent by having experimental subjects crossing their hands across the head-body midline.

\section{Experiment 3: Influence of "Response Bias" on Vestibular-Visual Prior Entry}

Angular transformation of individual percentages of "upfirst," "down-first," or "up-second," "down-second" responses corresponding to "left-first" responses to simultaneous trials were submitted to a Vestibular condition (motionless baseline, leftward rotation, rightward rotation) $\times$ by Type of judgment ("which came first," "which came second") within-subjects ANOVA. The ANOVA showed a significant main effect for vestibular condition $[F(2,24)=9, p=.001]$ with no Vestibular condition $\times$ Type of judgment interaction. Leftward accelerations increased the frequency of "left-first" responses (65.5\%) as compared with motionless baseline (57\%), whereas rightward accelerations decreased the frequency of "left-first" responses (48\%; all planned comparisons $p<.05$ ). Inspection of means shows that reporting which stimulus came second smoothed the difference between baseline and leftward rotation without reversing the pattern of responses observed under "which came first" instruction (see Figure 3). Percentages of "left-first" equivalent responses to asynchronous trials calculated in the whole sample and across the two instruction conditions are reported in Figure 3. At short asynchronies (from -15 to $+15 \mathrm{msec}$ ), rotatory accelerations produced directional biases similar to those observed for synchronous trials. Vestibular influences were not observed at longer asynchronies. Almost- 
Figure 2. Experiment 2: Vestibular-tactile prior entry. (A) Uncrossed hand condition: Percentage of "hand on left side first" responses in the three rotatory conditions and the VOR and no-VOR suppression conditions. Vertical bars inside line graphs indicate 95\% confidence interval (Loftus \& Masson, 1994). Percentage differences normalized to motionless results are reported inside the boxes to the right of line graphs $(\mathrm{MB}=$ motionless baseline; LR = leftward rotation; $\mathrm{RR}=$ rightward rotation). (A1) Uncrossed hand condition: Percentage of "left-first" responses to asynchronous trials (ordinate) plotted against asynchronies (abscissa).

Negative asynchronies indicate "first left-second right" trials, positive asynchronies "first right-second left" ones. (B) Crossed hand condition: Percentage of "hand on left side first" responses in the three rotatory conditions and in VOR and no-VOR suppression trials. (B1) Crossed hand condition: Percentage of "left-first" responses to asynchronous trials (ordinate) plotted against asynchronies (abscissa).

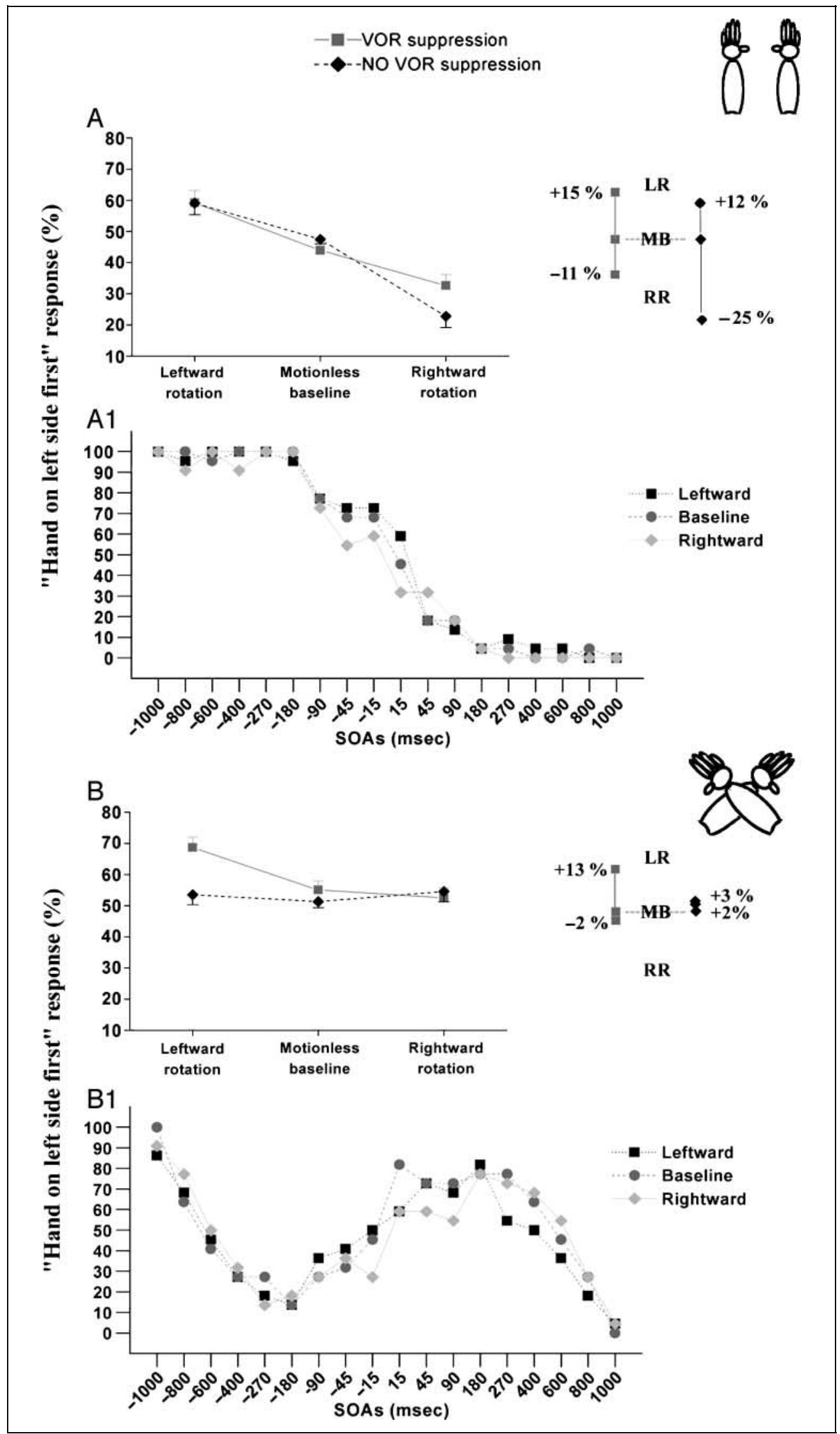


Figure 3. Experiment 3: Influence of "response bias" on vestibular-visual prior entry. (A) Percentage of "up-first," "down-first," "up-second," and "downsecond" responses equivalent to "left-first" responses, in the three rotatory and the two instruction conditions (i.e., "which came first" and "which came second"). Only VOR suppression condition was used in this experiment. Vertical bars inside line graphs indicate $95 \%$ confidence interval (Loftus \& Masson, 1994). Percentage differences normalized to motionless results are reported inside the box to the right of the line graph $(\mathrm{MB}=$ motionless baseline; LR = leftward rotation; $\mathrm{RR}=$ rightward rotation). (B) Percentage of "left-first" equivalent responses to asynchronous trials (ordinate) plotted against asynchronies (abscissa).

Negative asynchronies indicate "first left-second right" trials, positive asynchronies "first right-second left" ones.

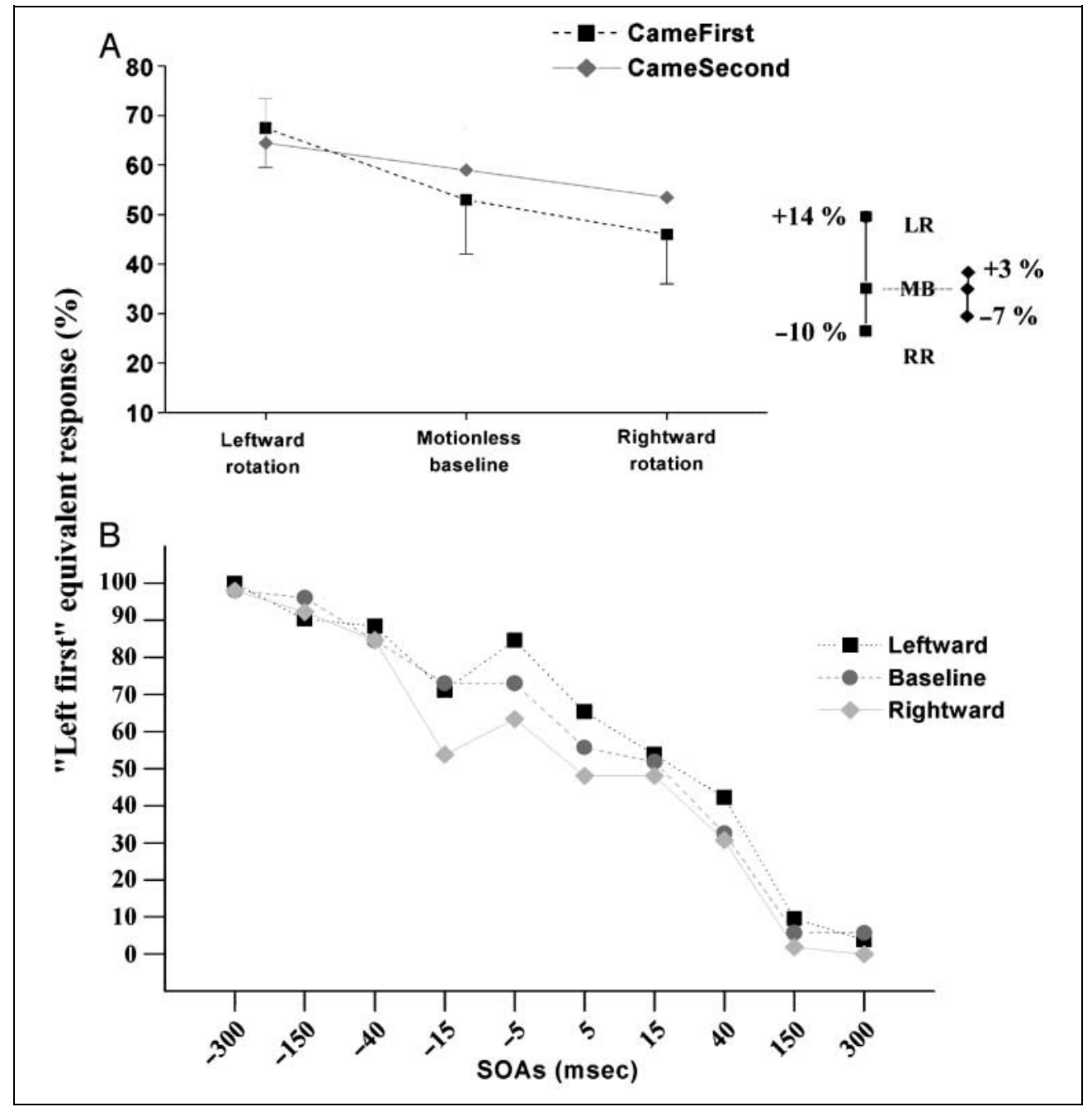

perfect performance was reached at the longest asynchrony ( + or $-300 \mathrm{msec}$ ).

These results suggest that attentional facilitatory effects caused by rotatory acceleration are still present even when the spatial coding of the vestibular stimulus is directionally dissociated from the spatial coding of the response required by the attentional task. This rules out relevant influence of response bias due to spatial compatibility between the directions of rotation and the directional labels used in TOJs.

\section{DISCUSSION}

We found that variations of vestibular inputs due to passive accelerations around the vertical head-body axis orient attention toward the direction of rotation and facilitate the detection of visual and tactile inputs presented on the side of rotation relative to those presented against the side of rotation. Vestibular facilitatory effects were present also when experimental subjects actively suppressed the VOR. This is in keeping with the hypothesis that covert orienting of attention might take place when a central motor plan is activated and, at the same time, its overt motor performance inhibited (Rizzolatti, Fogassi, \& Gallese, 1997).

Head-body rotations occurring in total darkness around the vertical body axis result in alternating slow and rapid eye movements, respectively, directed away and toward the sense of rotation. Slow movements tend to maintain gaze on spatial positions being left by rotation and, accordingly, are defined "compensatory." By contrast, fast movements directed toward the side of rotation are defined "anticompensatory" (Vidal, Berthoz, \& Milanvoye, 1982; Chun \& Robinson, 1978; Mellvill Jones, 1964). Our study first clarifies that during passive rotatory head-body accelerations, covert orienting of attention shifts toward the side of space where the rotatory movement and the fast components of the VOR are directed. This finding dovetails with the proposal that the rapid phases of the VOR do not simply return gaze to the center of the orbit but rather move the eyes beyond this primary position, orienting the oculomotor response towards new "centers of interest" located in the space being reached by rotation. This causes a shift 
of the beating field (i.e., the orbital position of the fast components of the VOR) in the direction of the movement, favoring the early recognition of targets located where the head is directed (Siegler et al., 1998; Mellvill Jones, 1964). Although in our subjects we did not evaluate the shift of the beating field, the findings from the present study might suggest that during head-body rotations, the direction of covert attentional orienting is coincident with the direction of the shift of the beating field.

Much of the interest in investigating vestibular stimulation on attention comes from the study of patients affected by unilateral neglect. In humans, neglect is characterized by defective orienting toward stimuli in the space contralateral to a unilateral cerebral lesion causing, among others, ipsilesional bias in the TOJ task (Husain \& Rorden, 2003; Robertson, Mattingley, Rorden \& Driver, 1998). The lesion is most frequently located in the right hemisphere, comprising the right inferior parietal lobe (Doricchi \& Tomaiuolo, 2003; Mort et al., 2003; Vallar \& Perani, 1986) and the underlying white matter connecting parietal and frontal areas (Doricchi \& Tomaiuolo, 2003; Leibovitch et al., 1998; Gaffan \& Hornak, 1997). Because neglect can appear independently of primary sensory or motor deficits, it was proposed that the main pathophysiological cause of the syndrome is the functional disruption in the damaged hemisphere of the neural network underpinning the multimodal coding of contralesional space. This interpretation finds support in the observation that cold caloric vestibular stimulation of the left ear, causing VOR with slow phases directed contralesionally (leftward), produces transient amelioration of visuospatial and somatosensory symptoms associated with neglect (Geminiani \& Bottini, 1992; Cappa, Sterzi, Vallar, \& Bisiach, 1987; Rubens, 1985; Marshall \& Maynard, 1983). This amelioration also includes apparently elementary deficits such as left hemianesthesia (Vallar, Bottini, \& Sterzi, 2003; Vallar, Bottini, Rusconi, \& Sterzi, 1993). These findings could be taken as evidence that appropriate variations of vestibular input, simulating headbody rotation toward the side of the lesion (rightward) and inducing slow phases of the VOR in the contralesional one (leftward), restore the allocation of attention toward the neglected left hemispace. By showing that attentional orienting in the visual and tactile modalities is biased toward the direction of head-body rotation and opposed to the direction of the slow phases of the VOR (i.e., toward the direction of fast phases), the data from the present study do not agree with an attentional account of neglect reduction following caloric vestibular stimulation (Vallar, Guariglia, \& Rusconi, 1997). Two alternative hypotheses can be advanced to explain caloric effects. First, it should be noted that in neglect patients caloric stimulations are usually administered in naturally lighted environments. This might cause the triggering of the leftward optokinetic response synergic to rightward head-body rotation. It is well known that visual, somatosensory, and auditory neglect can be reduced by optokinetic stimulation directed leftward (Pizzamiglio, Frasca, Guariglia, Incoccia, \& Antonucci, 1990). Therefore, secondary optokinetic effects induced by vestibular stimulation could mediate amelioration of neglect after caloric stimulation. Another account derives from the central effects of caloric stimulations documented by brain imaging studies showing the existence of cortical areas receiving afferent information from vestibular organs. In humans, cold water irrigation of one ear typically activates the temporo-parietal cortex, the insula, the putamen, and the anterior cingulate in the contralateral hemisphere (Bottini, Sterzi, et al., 1994). This "vestibular cortical system" not only receives afferent vestibular inputs, but also modulates ocular vestibular responses through efferent connections the to vestibular nuclei (Doricchi, Siegler, Iaria, \& Berthoz, 2002; Ventre-Dominey, Vighetto, \& Denise, 1999). A PET study by Bottini, Paulesu, et al. (1995) suggests that in right brain-damaged patients cold caloric vestibular stimulation reduces contralesional somatosensory deficits (i.e., hemianesthesia) by activating spared secondary somatosensory cortices where both tactile and vestibular signals are received (Johansen-Berg, Christensen, Woolrich, \& Matthews, 2000). This finding points out that remission of neglect following peripheral caloric stimulation of the left ear might depend on activation of cortical structures receiving afferent vestibular inputs in the right hemisphere. These structures are functionally commingled and anatomically close to those subserving multimodal space representations lateralized in the right hemisphere, so that activation of the former can temporarily restore activity of the latter.

Rorden, Karnath, and Driver (2001) found no effect of cold caloric vestibular stimulation of the left ear on covert visual orienting in normal subjects. These authors proposed that remission of neglect after caloric stimulation should be interpreted as deriving from changes induced in the representation of the egocentric space rather than from shifts of attention. According to the same authors, in humans, vestibular input could modulate the activity of cells similar to those in the posterior parietal cortex of the monkey (area 7b; Snyder, Grieve, Brotchie, \& Andersen, 1998), whose discharge to visual stimuli is modulated by vestibular inputs linked to trunk rotations. At variance with the study of Rorden et al., our findings demonstrate vestibular influence on covert attentional orienting. However, we agree with the conclusions of these authors, because shifts of covert orienting induced by rotatory vestibular stimulations were directionally opposed to what was expected on the basis of the effects of caloric vestibular stimulation in neglect patients. Discrepant results between our study and that of Rorden and co-workers might be due to several procedural differences. One possible difference is that the caloric vestibular stimulation of a single ear canal 
used by Rorden and co-workers did not cause the same level of vestibular input as the bilateral stimulation induced by head-body rotations in the present study. A second possible difference is that, at variance with the caloric stimulation used in the study or Rorden et al., the computer-driven rotatory movements used in the present study allowed for the precise synchronization between the dynamic properties of vestibular stimulation (i.e., the midpoint of the acceleration phase) and the presentation of stimuli. Finally, a third most relevant difference is that in our study the use of manual response keys placed along the direction of rotation (to the left or to the right of the head-body midsagittal plane) might have introduced a response motor bias (i.e., the tendency to select the motor response on the side of rotation; Shore, Spence, et al., 2001). This was absent in Rorden and co-workers' study, in which simple reaction times to lateralized visual stimuli were recorded through a central response button. In the first and second experiments of the present study, asking subjects to be accurate rather than quick caused long reaction times and absence of spatial compatibility effects between hand position and the lateral direction of rotation. This initially seemed to exclude a relevant influence of response bias. The results of the third control experiment, in which vestibular directional cueing and response dimensions were spatially dissociated (Shore, Spence, et al., 2001; Cairney, 1975; Drew, 1896), confirmed attentional prioritization of stimuli on the side of rotation. Stimuli presented on the side of rotation were still judged to come first as compared with simultaneous stimuli on the opposite side, even when the response label and the position of response buttons (i.e., "up" or "down") were both orthogonal to the direction of rotation (i.e., "left" or "right"). This suggests that facilitatory effects induced by vestibular stimulation affect perceptual processing rather than entering into play only at a later postperceptual response selection level (Shore, Spence, et al., 2001; Pashler, 1998). It should be cautiously noted, however, that in the third control experiment, prioritization effects seemed somewhat smaller as compared with the first experiment. This might indicate that vestibular influences on postperceptual processing amplify vestibular facilitation of perceptual processing. Importantly, in the control experiment, asking subjects to report which stimulus came "second" rather than "first" did not reverse prioritization of stimuli presented on the side of rotation. This further emphasizes that subjects did not simply report the stimulus presented on the side of rotation (i.e., response bias): If this was the case, then "which-came-second" instructions should have just reversed prioritization effects observed under "which-came-first" instructions (Shore, Spence, et al., 2001; Frey, 1990).

Our findings might provide an attentional account of some experimental results reported by Lewald and Karnath $(2000,2001)$. These authors found that the position of a binaural sound perceived straight ahead in the motionless condition is subjectively shifted in the direction of rotation during acceleratory head-body turns. Based on our findings, which show a reflexive attentional shift in the direction of rotation, we interpret the effect described by Lewald and Karnath $(2000,2001)$ as due to the fact that the monoaural sound on the side of rotation is attentionally prioritized relative to the one on the side opposite rotation. Attentional prioritization might create the illusion of an interaural difference between two identical and simultaneous monoaural stimuli, so that the stimulus on the side of rotation is perceived as preceding the contralateral one, as if it was arriving from a closer sound source. As a consequence, the subjective position of the resulting binaural stimulus seems to be shifted toward the side of space where the subjectively closer monoaural sound is localized.

The results of the second experiment showed that crossing upper limbs considerably reduced or eliminated the attentional advantage seen for tactile stimuli delivered to the hand lying on the side of rotatory acceleration in the uncrossed anatomical condition. This is in keeping with previous findings demonstrating that arm crossing reduces cross-modal facilitatory effects (see for tactile-visual interaction, Kennet, Eimer, Spence, \& Driver, 2001). Some evidence also demonstrates that arm crossing interferes with the correct perception of simultaneous bimanual stimuli in unilateral brain-damaged patients (Bartolomeo, Perri, \& Gainotti, 2004; Vaishnavi, Calhoun, \& Chatterjee, 2001; Aglioti, Smania, \& Peru, 1999) and with TOJs to asynchronous tactile stimuli in normal subjects (Shore, Spry, \& Spence, 2002; Yamamoto \& Kitazawa, 2001). When limbs are crossed, normal subjects can even experience subjective reversal in the temporal order of stimuli released at very short asynchrony intervals (Yamamoto \& Kitazawa, 2001). It has been proposed that with limbs crossed, time is needed to remap the position of hands in space using proprioceptive and efference copy signals from the arms to resolve the conflict between spatiotopic and somatotopic coding of stimulus position. At short interstimulus intervals (i.e., $<300 \mathrm{msec}$ ), there is not enough time for this process to be accomplished, causing subjective reversal in the temporal order of hand stimulation (Yamamoto \& Kitazawa, 2001): This finding was exactly replicated in the present study (see Figure 2 ). With limbs crossed, on synchronous trials some interesting differences emerged between rotations with and without VOR suppression. When VOR was not suppressed and a central fixation point was not available during rotation, no detectable difference in attentional bias was found among the motionless condition and the leftward or rightward turns. We argue that in the absence of the spatial reference provided by the central fixation point, the resolution of the conflict between spatiotopic and somatotopic coding of stimuli presented during headbody rotations is so difficult that vestibular facilitatory 
attentional effects are ineffective or cancelled out. When a central fixation reference was available to subjects and VOR was suppressed during rotation, facilitatory effects for stimuli released to the right hand lying in the left hemispace were observed during leftward rotatory accelerations. Importantly, this finding first suggests spatiotopic rather than somatotopic influences of vestibular inputs, indicating that cross-modal vestibular facilitatory effects operate on a representation of space that is updated following arm crossing. Similar effects were documented in tactile-visual interaction, in which covert visual orienting is improved on the side of space where the stimulated hand lies rather than for stimuli presented to the hemisphere the stimulated hand is directly anatomically connected with (Macaluso, Frith, \& Driver, 2002; Kennet, Eimer, Spence, \& Driver, 2001). These congruent findings seem to emphasize that tactile inputs are systematically remapped before being integrated with inputs from other modalities in the control of covert attentional orienting (however, see slightly different results for overt orienting in Groh \& Sparks, 1996).

Some convergent evidence may tentatively help clarify why in the crossed-arm condition with VOR suppression spatiotopic facilitatory vestibular effects were only observed for the right hand positioned in the left hemispace. In a recent fMRI study, Lloyd, Shore, Spence, and Calvert (2003) found that in subjects with eyes closed (i.e., an experimental condition well corresponding to the total darkness condition adopted in the present study which, independently of the presence of the central fixation point, did not allow subjects to see their limbs), tactile stimuli presented to the right hand positioned in the left hemispace selectively activated the ventral section of the intraparietal sulcus in the right hemisphere. Stimuli presented to the left hand positioned in the right hemispace caused activation of the same area in both hemispheres. In the present study, leftward rotatory accelerations caused leftward shift of attention. The attentional advantage seen for stimuli to the right hand in the left hemispace might therefore originate from the temporally and anatomically coincident activation of the network allocating attention toward the left hemispace in the right hemisphere (Gitelman et al., 1999; Nobre et al., 1997) and the network devoted to the proprioceptive coding of right hand positioning in the left hemispace, localized by Lloyd, Shore, Spence, and Calvert (2003) in the same hemisphere. Note also that the preferential engagement of the right parietal cortex in the perception of bimanual stimuli (Oliveri et al., 1999) might have further amplified the above-mentioned facilitatory effects. During rightward rotations, rightward shifts of attention might have preferentially engaged the left hemisphere (Mesulam, 2002). However, in this case, the poor hemispherically lateralized representation of crossed lefthand position and the minor competence of the left hemisphere in the detection of bimanual stimuli might have disfavored the summation of facilitatory effects similar to those putatively in action in the case of the right hand.

To conclude, our study provides evidence that passive rotary-vestibular accelerations influence covert orienting of attention. These original findings await replication and extension to the study of attentional orienting during active head-body movements.

\section{METHODS}

\section{Experimental Procedure}

\section{General}

A pair of stimuli was presented in each trial. In rotatory trials, stimuli were presented during the acceleratory phase of a passive angular movement given around the vertical head-body axis. The rotatory movement had a triangular profile with an initial 3-sec acceleratory phase followed by a 3-sec deceleratory one (total duration of rotation $=6 \mathrm{sec}$; frequency $=0.167 \mathrm{~Hz}$; amplitude $=324^{\circ}$; peak velocity $=108^{\circ} / \mathrm{sec}$; acceleration $=36^{\circ} / \mathrm{sec}^{2}$ ). Stimuli were presented at the midpoint of the acceleratory phase, $1500 \mathrm{msec}$ after the beginning of chair rotation (see Figure 4). A brief acoustic warning signal (500 msec) was delivered immediately before the start of rotation. In the baseline motionless condition, the same acoustic warning signal was delivered $1500 \mathrm{msec}$ before the presentation of experimental stimuli. Following a forced-choice paradigm, in each trial subjects were required to judge which stimulus came first by pushing the button placed on the side of the corresponding stimulus with their index finger. Subjects were told to concentrate on accuracy; we did not require speeded responses. By removing the pressure to respond quickly, this procedure should provide a more accurate index of the perceptual, rather than motor, components of information processing (Shore, Spence, et al., 2001).

In each experimental vestibular condition (motionless baseline, leftward acceleration, rightward acceleration), TOJs could be performed in two different oculomotor conditions. In the first oculomotor condition (no VOR suppression): (a) on rotatory trials, the LED at central fixation was on $2 \mathrm{sec}$ before the start of chair movement and off as rotation started (i.e., $1500 \mathrm{msec}$ prior to presentation of stimuli), allowing the release of the VOR; (b) on motionless trials, central fixation was off 1500 msec before presentation of stimuli and subjects were asked to maintain gaze straight ahead. In the second condition (VOR suppression), subjects were required to maintain gaze on the central fixation LED, which remained on for the entire duration of the trial. This allowed for the suppression of the VOR on rotatory trials. Trials in which eye movements were not properly suppressed (i.e., when horizontal eye movements greater than $1^{\circ}$ were observed before the presentation of 
Figure 4. (A) Top view of the experimental apparatus. Hands and response buttons positions in Experiments 1 and 2 (left/right responses) are in black. Hand and response buttons positions in Experiment 3 (up/down responses) are in gray. (B) Top: angular velocity profile of chair movement; middle: example of eye movements and velocity profile recorded in a "VOR suppression" trial; bottom: example of eye movements and velocity profile recorded in a "no-VOR suppression" trial.
A

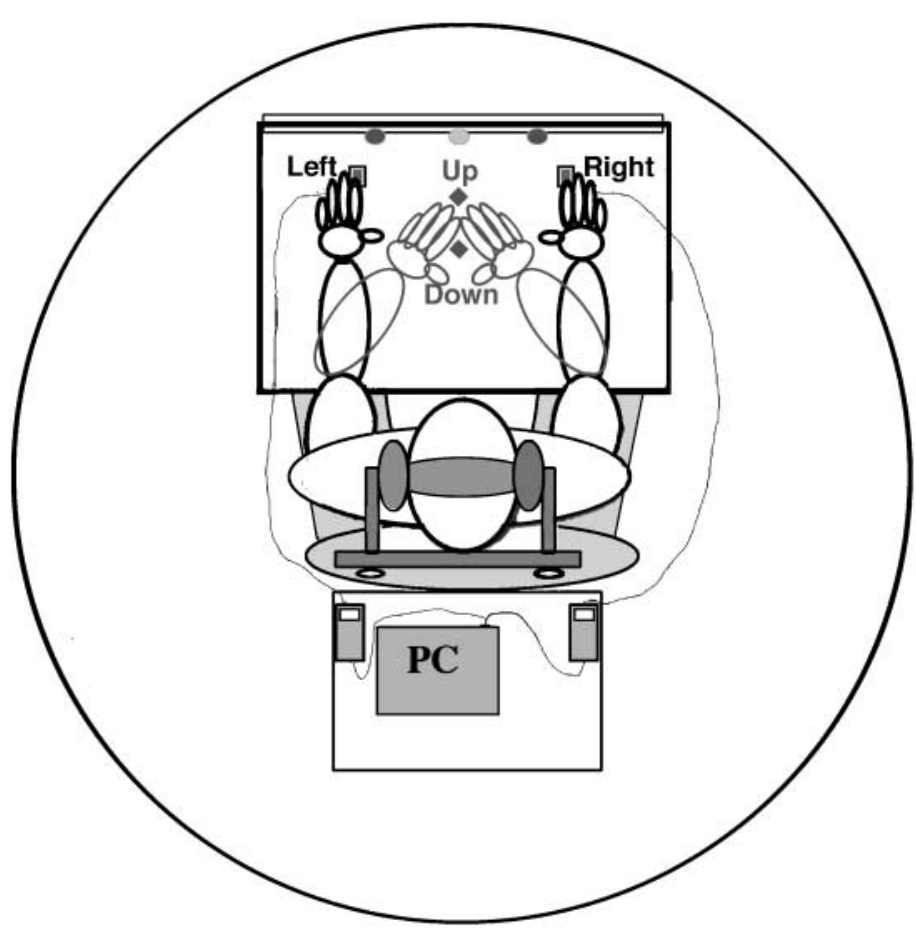

B
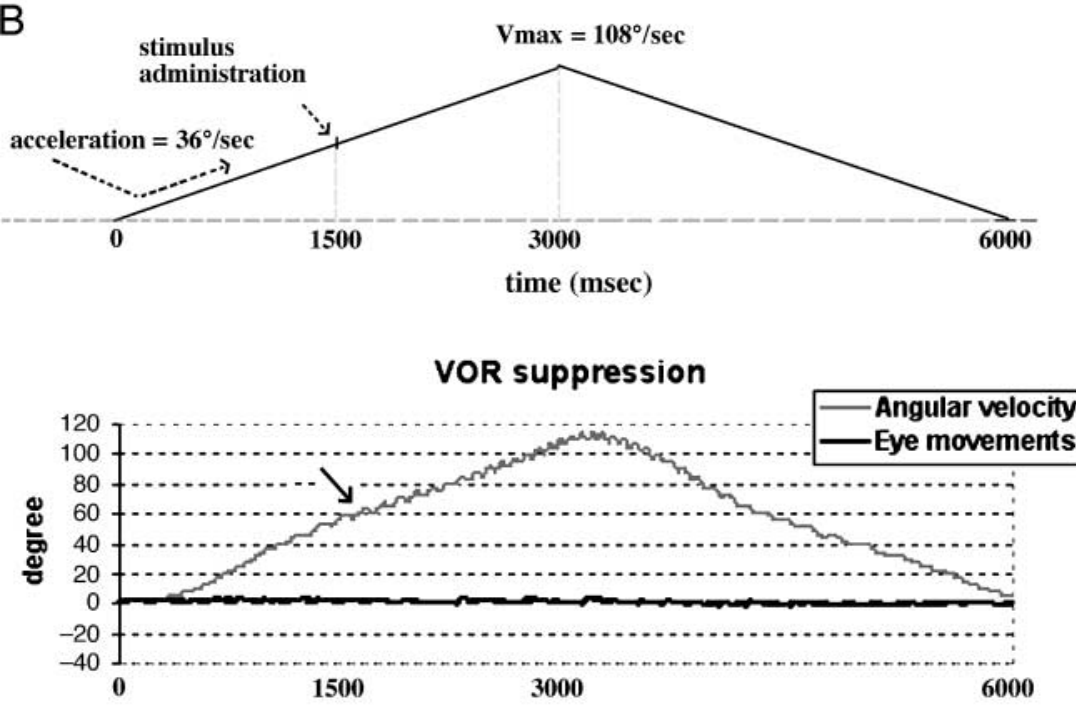

No VOR suppression

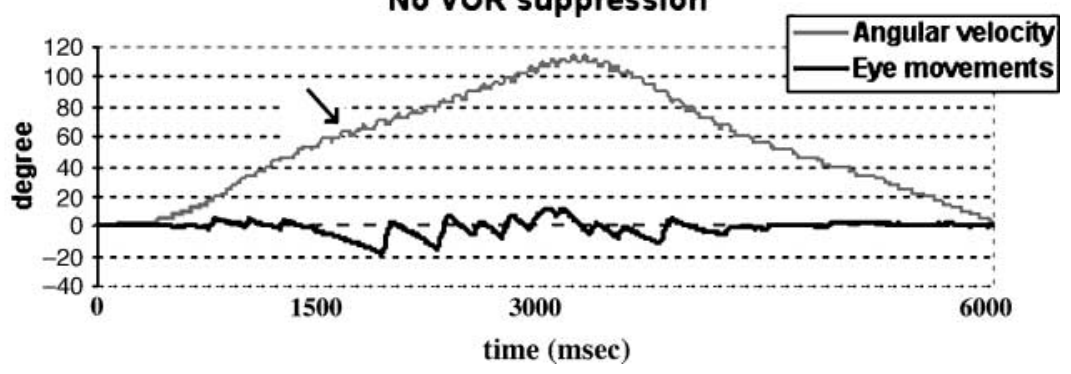


stimuli) were discarded and replaced at the end of the session. On average, few trials were replaced in each of the experiments (3-5\%).

The study of TOJ during rotatory accelerations requires appropriate modifications of the experimental procedure usually adopted in motionless conditions. The primary methodological problem is the need to interpose a suitable motionless time interval $(15 \mathrm{sec}$ in the present experiments) at the end of each rotatory trial (duration $=6 \mathrm{sec}$ ) to allow for the restabilization of the vestibular response in the semicircular canals. This means that the total duration of each trial is $21 \mathrm{sec}$ and implies a relevant increase in the time required for testing experimental subjects. We counteracted the overextension of testing time and the discomfort of the experimental subjects caused by repetitive rotatory head-body accelerations by reducing the number of experimental trials, using simultaneous trials as experimental ones and asynchronous trials as distracters. In Experiments 1 and 2, in each of the vestibular (motionless baseline, leftward acceleration, rightward acceleration) and experimental oculomotor conditions (VOR suppression, NO VOR suppression), 18 simultaneous and 18 asynchronous trials were administered. On half of the asynchronous trials, the left stimulus was delivered first (negative asynchrony), and on the other half of the trials (positive asynchrony), the reverse. Nine asynchrony intervals were used: $15,45,90,180,270,400$, 600, 800, and 1000 msec. In Experiment 3, in each of the vestibular conditions, 20 simultaneous and 20 asynchronous trials (10 with negative and 10 with positive asynchrony) were delivered. Based on the results from Experiment 1, showing perfect TOJs for asynchrony intervals greater than $270 \mathrm{msec}$, only five asynchrony intervals were used: 5, 15, 45, 180, and 300 msec. In each vestibular condition, each asynchrony interval was presented twice.

Variations in the frequency of "left-first" responses (Experiments 1 and 2) or "left-first" equivalent responses (Experiment 3) to simultaneous trials presented in each of the different rotatory and oculomotor conditions served as dependent variable. Individual proportions of "left-first" responses were submitted to angular (i.e., arcsin) transformation and entered in full-factorial repeated-measures ANOVAs. This transformation makes the variance of the binomial proportion independent from the probability of the binomial distribution (Zar, 1999), so that transformed data can be analyzed with statistical methods assuming normality and homogeneity of variance.

\section{Subjects and Stimuli}

All subjects were normal right-handed adults with no signs or history of vestibular disturbances. They voluntarily participated in the study. The experimental protocol was approved by the local ethical board.

\section{Experiment 1: Vestibular-Visual Prior Entry}

Eleven right-handed subjects (mean age $=26.8$ years, $S D=3$ ) participated in two sessions, one with and one without VOR suppression. The order of sessions was balanced among subjects. In each session, the lateral attentional bias in the "TOJ task" was first evaluated in the baseline-motionless condition and then during leftward and rightward head-body accelerations alternated in random order.

The brief $\left(5 \mathrm{msec} ; 2 \mathrm{~cd} / \mathrm{m}^{2}\right)$ above threshold illumination of two lateral red LEDs in complete darkness, one positioned $10^{\circ}$ to the left and the other $10^{\circ}$ to the right of the central fixation point, served as experimental stimuli.

\section{Experiment 2: Vestibular-Tactile Prior Entry}

Eleven right-handed subjects (mean age $=27.4$ years, $S D=3.8$ ) participated in the experiment. Four of these subjects also participated in Experiment 1. Subjects participated in four separate experimental sessions, two with VOR suppression (one with hands crossed and one with hands uncrossed) and two without VOR suppression (one with hands crossed and one with hands uncrossed). Order of sessions with and without VOR suppression was balanced among subjects. In each session, the lateral attentional bias in the "TOJ task" was first evaluated in the baseline-motionless condition and then during leftward and rightward head-body accelerations alternated in random order.

Tactile stimuli were nonnoxious electric shocks delivered by monophasic electric current stimulators (STM 140, High Technology Laboratory, Udine, Italy; electrodes $1 \mathrm{~mm}$ diameter) positioned on the dorsal surface of the second phalanx of the left and the right fourth finger. The intensity of the tactile stimulation was determined for each subject at the beginning of each experimental session. This procedure allowed us to deliver stimuli which were clearly detected and which were perceived with comparable and steady subjective intensity on both fingers. Mean intensity value was $3.3 \pm$ $4.7 \mathrm{~mA}$. The duration of tactile stimuli was $5 \mathrm{msec}$. The electric stimulators were controlled by an IBMcompatible computer.

\section{Experiment 3: Influence of "Response Bias" on Vestibular-Visual Prior Entry}

Thirteen right-handed subjects (mean age $=26.4$ years, $S D=3.1)$ participated in two experimental sessions. None of these subjects took part in the two previous experiments. Only the VOR suppression condition was used in this experiment. Four red LEDs served as stimuli (duration and intensity of stimulation were identical to those from Experiment 1). Two LEDs were placed $10^{\circ}$ to the left and two other LEDs $10^{\circ}$ to the right of 
the central fixation point. On each side, one LED was positioned $2^{\circ}$ above and the other LED $2^{\circ}$ below an horizontal bar (extension $1^{\circ}$ ) made with dimly illuminated green LEDs. One out of two combinations of stimuli were presented in each trial: "up-left/down-right" or "down-left/up-right." The two combinations were equally distributed in both simultaneous and asynchronous trials. In each session, the lateral attentional bias in the "TOJ task" was first evaluated in the baseline-motionless condition and then during leftward and rightward headbody accelerations alternated in random order. In one session, we asked subjects to decide whether the stimulus above or below the horizontal bar appeared first, and in the other session, whether the stimulus above or below the horizontal bar appeared second. This was done to check further the influence of response bias: If observers simply report the stimulus on the side of rotation, then TOJs should shift in opposite directions under the different "which-came-first" versus "whichcame-second" instructions (Shore, Spence, et al., 2001; Frey, 1990). The order of sessions was balanced among subjects. Subjects formulated their TOJs pressing one of two response buttons with both index fingers. Buttons were positioned on a horizontal panel and aligned along the head-body midsagittal plane. The upper button was used for "up-first" or "up-second" responses and the lower button for "down-first" or "down-second" responses (see Figure 4). Subjects were accurately instructed to disregard velocity of response and favor accuracy of response.

\section{Apparatus}

The experimental apparatus depicted in Figure 4 consisted of a motorized chair rotating around the vertical gravitational axis (mod. "Rotomac," Megaris s.a.s.). The software controlling the movement of the chair recorded and stored chair position (sampling rate $100 \mathrm{~Hz}$ ) with a resolution of $0.072^{\circ}$ of arc. Two panels were secured to the chair. On the anterior panel, there were two manual response buttons and a bar. The bar held a central green fixation LED aligned to the head-body midsagittal plane and the lateral red LEDs. The bar was parallel to the coronal plane of the head and positioned at a distance of $57 \mathrm{~cm}$ from the head of experimental subjects. LEDs were positioned at eye level and the response button was positioned at elbow level (considering upper arm aligned to the trunk). Subjects were tested in complete darkness. Illumination of LEDs did not allow subjects to see their arms.

The following items were positioned on the posterior panel: (a) a laptop controlling the delivery of stimuli and the recording of manual responses through E-Prime software; (b) two monophasic electric current stimulators (HTL mod. STM140) for the administration of tactile stimuli; (c) a DC amplifier for EOG recording. Horizontal and vertical EOG were recorded with electrodes placed at the outer canthi and above and below the right eye. In order to avoid the influence of brisk variations of the retinal-corneal dipole, experimental sessions started after a 15-min adaptation period to darkness. EOG calibration was run at the beginning of each session and checked again every four trials. The calibration procedure was run by asking subjects to fixate, in sequence, the left, the central, and the right LED. EOG signals were amplified, filtered, digitalized (sampling rate $200 \mathrm{~Hz}$ ), and stored for off-line processing.

Another PC controlled the movement of the chair and its synchronization with the delivering of stimuli, the $\mathrm{AD}$ conversion and storing of eye movement signals, the storing of the chair velocity profile, the recording of event markers indicating the state of LEDs (on/ off), and the release of tactile stimuli. During experimental trials, white noise masking spurious environmental sounds was delivered to experimental subjects through headphones.

\section{Acknowledgments}

This work was supported by grants from the Universita "La Sapienza"-Roma and the Fondazione Santa Lucia. We thank Prof. R. Ivry and two anonymous referees for useful suggestions on the manuscript. The invaluable technical contributions of P. Fermani, U. Cellini, and D. Moretti are also acknowledged.

Reprint requests should be sent to Prof. Fabrizio Doricchi, Centro Ricerche di Neuropsicologia, Fondazione Santa LuciaIRCCS, Via Ardeatina 306-00179 Rome, Italy, or via e-mail: Fabrizio.Doricchi@uniroma1.it.

\section{REFERENCES}

Aglioti, S., Smania, N., \& Peru, A. (1999). Frames of references for mapping tactile stimuli in brain-damaged patients. Journal of Cognitive Neuroscience, 11, 67-79.

Andersen, R. A., Snyder, L. H., Bradley, D. C., \& Xing, J. (1997). Multimodal representation of space in the posterior parietal cortex and its use in planning movements. Annual Review of Neuroscience, 20, 303-330.

Bartolomeo, P., Perri, R., \& Gainotti, G. (2004). The influence of limb crossing on left tactile extinction. Journal of Nerology, Neurosurgery, and Psychiatry, 75, 49-55.

Berthoz, A. (1997). Le sens du mouvement. Paris: Odile Jacob. Bottini, G., Sterzi, R., Paulesu, E., Vallar, G., Cappa, S. F., Erminio, F., Passingham, R. E., Frith, C. D., \& Frackowiak, R. S. J. (1994). Identification of the central vestibular projections in man: A position emission tomography activation study. Experimental Brain Research, 99, 164-169.

Bottini, G., Paulesu, E., Sterzi, R., Warburton, E., Wise, R. J., Vallar, G., Frackowiak, R. S. J., \& Frith, C. D. (1995). Modulation of conscious experience by peripheral sensory stimuli. Nature, 376, 778-781.

Bremmer, F., Schlack, A., Duhamel, J. R., Graf, W., \& Fink, G. R. (2001). Space coding in primate posterior parietal cortex. Neuroimage, 14, S46-S51.

Cairney, P. T. (1975). Bisensory order judgement and the prior entry hypothesis. Acta Psychologica, 39, 329-340. 
Cappa, S., Sterzi, R., Vallar, G., \& Bisiach, E. (1987). Remission of hemineglect and anosognosia during vestibular stimulation. Neuropsychologia, 25, 775-782.

Chun, K. S., \& Robinson, D. A. (1978). A model of quick phase generation in the vestibular-ocular reflex. Biological Cybernetics, 3, 209-221.

Clark, B., \& Graybiel, A. (1949). The effect of angular acceleration on sound localization: The audiogyral illusion. Journal of Psychology, 28, 235-244.

Crowell, J. A., Banks, M. S., Shenot, K. V., \& Andersen, R. A. (1998). Visual self-motion perception during head turns.

Nature Neuroscience, 1, 732-737.

Drew, F. (1896). Attention: Experimental and critical. American Journal of Psychology, 7, 533-572.

Doricchi, F., Siegler, I., Iaria, G., \& Berthoz, A. (2002). Vestibulo-ocular and optokinetic impairments in left unilateral neglect. Neuropsychologia, 40, 2084-2099.

Doricchi, F., \& Tomaiuolo, F. (2003). The anatomy of neglect without hemianopia: A key role for parietal-frontal disconnection? NeuroReport, 14, 2239-2243.

Driver, J., \& Spence, C. (1998). Attention and the crossmodal construction of space. Trends in Cognitive Sciences, 2, $254-261$.

Gaffan, D., \& Hornak, J. (1997). Visual neglect in the monkey. Representation and disconnection. Brain, 120, 1647-1657.

Galletti, C., Battaglini, P. P., \& Fattori, P. (1993). Parietal neurons encoding spatial locations in craniotopic coordinates. Experimental Brain Research, 96, 221-229.

Geminiani, G., \& Bottini, G. (1992). Mental representation and temporary recovery from unilateral neglect after vestibular stimulation. Journal of Neurology, Neurosurgery, and Psychiatry, 55, 332-333.

Gitelman, D. R., Nobre, A. C., Parrish, T. B., LaBar, K. S., Kim, Y. H., Meyer, J. R., \& Mesulam, M. (1999). A large-scale distributed network for covert spatial attention. Further anatomical delineation based on stringent behavioral and cognitive controls. Brain, 122, 1093-1106.

Graybiel, A., \& Hupp, D. (1946). The oculo-gyral illusion, a form of apparent motion which may be observed following stimulation of the semicircular canals. Journal of Aviation Medicine, 17, 3-27.

Graziano, M. S. A., Hu, X. T., \& Gross, C. G. (1997). Visuospatial properties of ventral premotor cortex. Journal of Neurophysiology, 77, 2268-2292.

Groh, J. M., \& Sparks, D. L. (1996). Saccades to somatosensory targets: I. Behavioral characteristics. Journal of Neurophysiology, 75, 412-427.

Israël, I., Bronstein, A. M., Kanayama, R., Faldon, M., \& Gresty, M. A. (1996). Visual and vestibular factors influencing vestibular "navigation". Experimental Brain Research, 112, 411-419.

Johansen-Berg, H., Christensen, V., Woolrich, M., \& Matthews, P. M. (2000). Attention to touch modulates activity in both primary and secondary somatosensory areas. NeuroReport, 11, 1237-1241.

Husain, M., \& Rorden, C. (2003). Non spatially lateralized mechanisms in hemispatial neglect. Nature Reviews: Neuroscience, 14, 26-36.

Kennett, S., Eimer, M., Spence, C., \& Driver, J. (2001). Tactile-visual links in exogenous spatial attention under different postures: Convergent evidence from psychophysics and ERPs. Journal of Cognitive Neuroscience, 13, 462-478.

Leibovitch, F. S., Black, S. E., Caldwell, C. B., Ebert, P. L., Ehrlich, L. E., \& Szalai, J. P. (1998). Brain-behavior correlations in hemispatial neglect using CT and SPECT: The Sunnybrook Stroke Study. Neurology, 50, 901-908.
Leigh, R. J., \& Zee, D. S. (1999). The vestibular optokinetic system. In R. J. Leigh \& D. S. Zee (Eds.), The neurology of eye movements (pp. 19-89). Oxford: Oxford University Press.

Lewald, J., \& Karnath, H. O. (2000). Vestibular influence on human auditory space perception. Journal of Neurophysiology, 84, 1107-1111.

Lewald, J., \& Karnath, H. O. (2001). Sound lateralization during passive whole-body rotation. European Journal of Neuroscience, 13, 2268-2272.

Lloyd, D. M., Shore, D. I., Spence, C., \& Calvert, G. A. (2003). Multisensory representation of limb position in human premotor cortex. Nature Neuroscience, 6, 17-18.

Loftus, G. R., \& Masson, M. E. (1994). Using confidence intervals in within-subjects designs. Psychonomic Bulletin $\mathcal{E}$ Review, 1, 476-490.

Macaluso, E., Frith, C. D., \& Driver, J. (2002). Crossmodal spatial influences of touch on extrastriate visual areas take current gaze direction into account. Neuron, 34, 647-658.

Marshall, C. R., \& Maynard, F. M. (1983). Vestibular stimulation for supranuclear gaze palsy: A case report. Archives of Physiology and Medicine Rehabilitation, 64, 134-136.

Mellvill Jones, G. (1964). Predominance of anti-compensatory oculo-motor response during rapid head rotation. Aviation, Space, and Environmental Medicine, 35, 965-968.

Meredith, M. A., \& Stein, B. E. (1986). Spatial factors determine the activity of multisensory neurons in cat superior colliculus. Brain Research, 19, 350-354.

Mesulam, M. M. (2002). Functional anatomy of attention and neglect: From neurons to networks. In H. O. Karnath, D. Milner, \& G. Vallar, (Eds.), The cognitive and neural bases of spatial neglect (pp. 33-46). Oxford: Oxford University Press.

Mort, D. J., Malhotra, P., Mannan, S. K., Rorden, C., Pambakian, A., Kennard, C., \& Husain, M. (2003). The anatomy of visual neglect. Brain, 126, 1986-1997.

Nobre, A. C., Sebestyen, G. N., Gitelman, D. R., Mesulam, M. M., Frackowiak, R. S., \& Frith, C. D. (1997). Functional localization of the system for visuospatial attention using positron emission tomography. Brain, 120, 515-533.

Oliveri, M., Rossini, P. M., Pasqualetti, P., Traversa, R., Cicinelli, P., Palmieri, M. G., Tomaiolo, F., \& Caltagirone, C. (1999). Interhemispheric asymmetries in the perception of unimanual and bimanual cutaneous stimuli. Brain, 122, 1721-1729.

Pashler, H. E. (1998). The psychology of attention. Cambridge: MIT Press.

Pizzamiglio, L., Frasca, R., Guariglia, C., Incoccia, C., \& Antonucci, G. (1990). Effect of optokinetic stimulation in patients with visuospatial neglect. Cortex, 26, 535-540.

Rizzolatti, G., Fogassi, L., \& Gallese, V. (1997). Parietal cortex: From sight to action. Current Opinion in Neurobiology, 7, 562-567.

Robertson, I. H., Mattingley, J. B., Rorden, C., \& Driver, J. (1998). Phasic alerting of neglect patients overcomes their spatial deficit in visual awareness. Nature, 395, 169-173.

Rorden, C., Karnath, H. O., \& Driver, J. (2001). Do neck-proprioceptive and caloric-vestibular stimulation influence covert visual attention in normals, as they influence visual neglect? Neuropsychologia, 39, 364-375.

Rubens, A. B. (1985). Caloric stimulation and unilateral visual neglect. Neurology, 35, 1019-1024.

Shore, D. I., Spence, C., \& Klein, R. M. (2001). Visual prior entry. Psychological Science, 12, 205-212.

Shore, D., Spry, E., \& Spence, C. (2002). Confusing the mind by crossing the hands. Cognitive Brain Research, 14, 153-163.

Siegler, I., Israël, I., \& Berthoz, A. (1998). Shift of the beating field of vestibular nystagmus: An orientation strategy? Neuroscience Letters, 254, 93-96. 
Snyder, L. H., Grieve, K. L., Brotchie, P., \& Andersen, R. A. (1998). Separate body- and world-referenced representations of visual space in parietal cortex. Nature, 27, 887-891.

Spence, C., Nicholls, M. E. R., Gillespie, N., \& Driver, J. (1998). Cross-modal links in exogenous covert spatial orienting between touch, audition, and vision. Perception $\&$ Psychophysics, 60, 544-557.

Spence, C., Shore, D. I., \& Klein, R. M. (2001). Multisensory prior entry. Journal of Experimental Psychology: General, 130, 799-832.

Stelmach, L., \& Herdman, C. M. (1991). Directed attention and perception of temporal order. Journal of Experimental Psychology: Human Perception and Performance, 17, 539-540.

Umiltà, C., \& Nicoletti, R. (1990). Spatial stimulus-response compatibility. In R. W. Proctor \& T. G. Reeve (Eds.), Stimulus-response compatibility: An integrated perspective (pp. 89-116). Amsterdam: North-Holland.

Vaishnavi, S., Calhoun, J., \& Chatterjee, A. (2001). Binding personal and peripersonal space: Evidence from tactile extinction. Journal of Cognitive Neuroscience, 13, 181-189.

Vallar, G., Bottini, G., \& Sterzi, R. (2003). Anosognosia for left-sided motor and sensory deficits, motor neglect, and sensory heminattention: Is there a relationship? Progress in Brain Research, 142, 289-301.

Vallar, G., Bottini, G., Rusconi, M. L., \& Sterzi, R. (1993).
Exploring somatosensory hemineglect by vestibular stimulation. Brain, 116, 71-86.

Vallar, G., Guariglia, C., \& Rusconi, M. L. (1997). Modulation of the neglect syndrome by sensory stimulation. In P. Their \& H. O. Karnath (Eds.), Parietal lobe contribution to orientation in 3 D space (pp. 555-578). Heidelberg: Springer.

Vallar, G., \& Perani, D. (1986). The anatomy of unilateral neglect after right-hemisphere stroke lesions. A clinical/CT-scan correlation study in man. Neuropsychologia, 24, 609-622.

Ventre-Dominey, J., Vighetto, A., \& Denise, P. (1999). Vestibular-ocular dysfunction induced by cortical damage in man: A case report. Neuropsychologia, 37, 715-721.

Vidal, P. P., Berthoz, A., \& Milanvoye, M. (1982). Difference between eye closure and visual stabilization in the control of the posture in man. Aviation, Space, and Environmental Medicine, 53, 1966-1967.

Vuilleumier, P., \& Schwartz, S. (2001). Modulation of visual perception by eye gaze direction in patients with spatial neglect and extinction. NeuroReport, 12, 2101-2104.

Wallace, M. T., Meredith, M. A., \& Stein, B. E. (1992). Integration of multiple sensory modalities in cat cortex. Experimental Brain Research, 91, 484-488.

Yamamoto, S., \& Kitazawa, S. (2001). Reversal of subjective temporal order due to arm crossing. Nature Neuroscience, 4, 759-765.

Zar, J. H. (1999). Biostatistical analysis. Upper Saddle River, NJ: Prentice Hall. 